\title{
Bone and Mineral Metabolism
}

\author{
Dov Tiosano \\ Meyer Children's Hospital, Rambam Medical Center, Haifa, Israel
}

\section{Calcium metabolism Calcium absorption}

\section{Relationships among vitamin D levels, parathyroid hormone, and calcium absorption in young adolescents}

\author{
Abrams SA, Griffin IJ, Hawthorne KM, Gunn SK, Gundberg CM, Carpenter TO \\ United States Department of Agriculture/Agricultural Research Service, Children's Nutrition Research Center, \\ Houston, Tex., USA \\ sabrams@bcm.edu \\ J Clin Endocrinol Metab 2005:90:5576-5581
}

Background: Evidence suggests that vitamin D status in adults is positively associated with calcium absorption fraction and inversely associated with serum PTH. The objective of this study was to evaluate the relationships among vitamin D status, PTH, and calcium absorption in midpubertal boys and girls.

Methods: Calcium absorption was measured by using a stable isotope method in 93 young adolescents, $12.7 \pm 1.0$ years of age, receiving diets averaging approximately $900 \mathrm{mg} /$ day calcium.

Results: A significant positive relation to calcium absorption was found for serum 1,25-dihydroxyvitamin $\mathrm{D}(\mathrm{p}=0.048)$ and PTH $(\mathrm{p}=0.007)$, but not for $25-\mathrm{OHD}(\mathrm{p}=0.77)$. PTH was significantly inversely related to $25-\mathrm{OHD}$ and was positively related to serum 1,25-dihydroxyvitamin D and osteocalcin. PTH was marginally significantly inversely related to lumbar spinal, but not whole body, bone mineral density. Conclusions: These data suggest that in adolescents, especially in the presence of vitamin D insufficiency, PTH secretion increases to adapt to higher rates of bone formation associated with growth. This results in higher serum $1,25(\mathrm{OH})_{2} \mathrm{D}$ concentrations and increased calcium absorption results.

The authors examined the correlation between calcium absorption and 25-OHD levels in adolescence, and found no relation between the two. However, when the patients were divided into subgroups, it was found that below the $25-\mathrm{OHD}$ level of $25 \mathrm{ng} / \mathrm{ml}(52 \mathrm{nmol} / \mathrm{l})$ there was a significant inverse correlation between calcium absorption and serum 25-OHD. In previous studies in adults, optimal 25-OHD levels were found to be $>23 \mathrm{ng} / \mathrm{ml}$, and in this study the minimum threshold desired for adolescence is $25 \mathrm{ng} / \mathrm{ml}$. When 25-OHD levels are lower, PTH rises and stimulates the synthesis of $1,25-\mathrm{OH}_{2} \mathrm{D}$ to accelerate calcium absorption. The definition of vitamin $\mathrm{D}$ insufficiency should be adjusted to this new threshold and the prevailing $15 \mathrm{ng} / \mathrm{ml}$ level that we have used so far should be abandoned. Concerning bone density, the authors found a marginally significant inverse relationship between PTH and lumbar spine bone mineral density. This suggests that this region of cuboid bone osteogenesis is the first to be affected by vitamin D insufficiency. 


\section{Height and height Z-score are related to calcium absorption in 5- to 15-year-old girls}

Abrams SA, Griffin IJ, Hawthorne KM, Liang L

United States Department of Agriculture/Agricultural Research Service,

Children's Nutrition Research Center, Houston, Tex., USA

sabrams@bcm.edu

J Clin Endocrinol Metab 2005;90:5077-5081

Background: Understanding the relationship between calcium absorption and growth has been limited. The aim of the study was to investigate the relationship between calcium absorption and height in prepubertal and pubertal girls.

Methods: In 315 girls aged 5.0-15.0 years the relationship between height, its age- and gender-normalized value (height $\mathrm{Z}$-score), and calcium absorptive efficiency were assessed.

Results: Overall, height was significantly related to calcium absorption (corrected for calcium intake, age, Tanner, stage, and ethnicity) $(\mathrm{p}=0.001)$. Similarly, height $Z$-score was significantly related to calcium absorption $(\mathrm{p}<0.007)$. Calcium absorption was significantly lower in girls with height $Z$-score $\leq 0$ compared with those with a height $Z$-score $>0$ (difference $3.9 \pm 1.4 \%$, mean \pm SEM; $p=0.007$ ). Conclusion: These results indicate that a small but significant component of the variability in calcium absorption is related to height. Identifying genetic risk factors for lowered calcium absorption during growth could lead to individual approaches for prevention of inadequate bone mass.

The significant correlation between height and calcium absorption in girls throughout all stages of puberty calls for further investigation into the factors that influence both height and calcium absorption. We know of the correlation between vitamin D receptor polymorphism, calcium absorption, and bone mineralization. But how does calcium absorption influence the growth plate growth and differentiation? An explanation is provided by a publication this year that extracellular calcium levels modulate the pace of growth plate chondrocyte differentiation [1], which comes as an important additive to last year's findings that phosphorus is essential for hypertrophic cell apoptosis. The question of whether calcium absorption is related to height and body size is present only during the growth period or whether it continues into adulthood is addressed in the next article. It turns out that body size and calcium absorption share similar regulatory mechanisms.

\section{Calcium absorptive efficiency is positively related to body size}

Barger-Lux MJ, Heaney RP

Creighton University, Omaha, Nebr., USA

J Clin Endocrinol Metab 2005;90:5118-5120

Background: Calcium absorption efficiency is a more important determinant of calcium balance than calcium intake itself. The sources of variability in absorptive performance are only partly elucidated. The aim of the study was to explore the relationship between body size and calcium absorption efficiency. Methods: 178 women, with an average age of 50.2 years, were studied from one to five times and yielded an aggregate data set containing 633 individual studies.

Results: Absorption residuals were significantly positively correlated with height, weight, and surface area, and after adjusting for estrogen status, these body size variables accounted for approximately $4 \%$ of the total variability.

Conclusion: The magnitude of the effect is such that a woman $1.8 \mathrm{~m}$ in height would absorb $30 \%$ more calcium from a given intake than a woman $1.4 \mathrm{~m}$ tall.

Calcium absorption is normally identical to calcium excretion and they both amount to about $200 \mathrm{mg} /$ day in an adult. What is the cause and what is the effect? We now learn that larger women absorb more calcium than smaller ones. This may constitute a part of the physiological mechanism for their tendency to have greater bone masses, which may account for the inclination of tall individuals to have more urinary calcium excretion. If smaller women absorb $30 \%$ less calcium, they may 
need greater amounts of calcium supplements to maintain adequate calcium levels and match up with urinary calcium loss. More on calcium metabolism and growth in the following article:

\section{Maternal 25-hydroxyvitamin D and parathyroid hormone concentrations and offspring birth size}

Morley R, Carlin JB, Pasco JA, Wark JD

Clinical Epidemiology University of Melbourne Department of Pediatrics, Royal Children's Hospital,

Parkville, Vic., Australia

morleyr@unimelb.edu.au

J Clin Endocrinol Metab 2006;91:906-912

Background: There is inconsistent evidence that maternal 25 -hydroxyvitamin $\mathrm{D}(25-(\mathrm{OH}) \mathrm{D})$ deficiency may impair fetal growth. The aim of this study was to examine the relationship between maternal $25-(\mathrm{OH}) \mathrm{D}$ and parathyroid hormone concentrations at $<16$ and 28 weeks of gestation and offspring birth size.

Methods: 475 women were enrolled in the study.

Results: $374 / 475$ (79\%) women completed this study. There was no evident relationship between birth size measures and maternal 25-(OH)D or PTH at recruitment (approx. 11 weeks). Gestation length was 0.7 weeks shorter $(95 \% \mathrm{CI}-1.3,-0.1)$ and knee-heel length $4.3 \mathrm{~mm}$ smaller $(-7.3,-1.3)$ in infants of 27 mothers with low $25-(\mathrm{OH}) \mathrm{D}(<28 \mathrm{nmol} / \mathrm{l})$ at $28-32$ weeks versus babies whose mothers had higher concentrations. This latter difference was reduced to $-2.7 \mathrm{~mm}(-5.4,-0.1)$ after adjustment for duration of gestation, suggesting some of the apparent growth deficit is explained by a shorter gestation. There was no evidence that other birth measures were affected. Maternal PTH concentration at 28-32 weeks was positively related to knee-heel length, birth weight and mid-upper arm and calf circumferences. These associations were independent of $25-(\mathrm{OH}) \mathrm{D}$ concentration.

Conclusions: Low maternal $25-(\mathrm{OH}) \mathrm{D}$ in late pregnancy is associated with reduced intrauterine long bone growth and slightly shorter gestation.

This article presents another link between growth, body size and vitamin D. From previous works it is known that maternal calcium concentration falls during pregnancy as the fetus grows. In this study the authors show that if low levels of 25-OHD are present when calcium levels decrease during late pregnancy (gestational weeks 28-32), the pregnancy gets shorter and growth of the fetal long bones is compromised. Although no significant relationship was found between $25-\mathrm{OHD}$ levels at the beginning of pregnancy and its duration or the fetus size at birth, it is recommended that women of childbearing age have $25-\mathrm{OHD}$ levels $>28 \mathrm{ng} / \mathrm{ml}(60 \mathrm{nmol} / \mathrm{l})$ from the start of the pregnancy and throughout its duration. The recommendation for calcium intake by pregnant women over the age of 18 is $1,000 \mathrm{mg}$ calcium per day and $1,300 \mathrm{mg}$ calcium per day under the age of 18 . The extended next review addresses the issue of recommendation for vitamin $D$ supplements.

\section{Reviews}

\section{The vitamin D questions: how much do you need and how should you get it?}

Wolpowitz D, Gilchrest BA

Department of Dermatology, Boston University School of Medicine, Boston, Mass., USA

J Am Acad Dermatol 2006;54:301-317

Summary: UV radiation is a well-documented human carcinogen, indisputably linked to the current continued increased rate of skin cancer. UV radiation is also responsible for cutaneous synthesis of vitamin $\mathrm{D}_{3}$, a substance that is then sequentially hydroxylated in the liver and kidney to yield $1,25(\mathrm{OH})_{2}$ vitamin D, a hormone critical for calcium homeostasis and skeletal maintenance. Because the UV action spectra for DNA damage leading to skin cancer and for vitamin D photosynthesis are virtually identical, 
the harmful and beneficial effects of UV irradiation are inseparable. This has given rise to the argument that sun avoidance, with a goal of skin cancer prevention, may compromise vitamin D sufficiency. Public interest in this matter has been heightened in recent years by multiple studies correlating the level of $25-\mathrm{OH}$ vitamin $\mathrm{D}$, the readily measurable 'storage' precursor form of the vitamin, with a variety of benefits separate from skeletal health. Although the studies are of variable quality and all alleged treatment benefits are based on dietary supplementation with vitamin D, not on increased sun exposure, they have been interpreted by some as support for advocating increased sun exposure of the public at large. The goal of this review is to provide a detailed, balanced, and referenced discussion of the complex literature underlying the current popular interest in vitamin D and sun exposure for the purpose of increasing vitamin D photosynthesis.

This comprehensive review provides a detailed, balanced, and referenced discussion of the complex literature underlying the current popular interest in vitamin $D$ and sun exposure for the purpose of increasing vitamin D photosynthesis. The authors review the nomenclature, metabolism, and established functions of vitamin D; the evidence supporting the less well-established but purported vitamin $D$ effects; the concept of vitamin $D$ insufficiency; populations at risk for vitamin $D$ deficiency, and finally the risk/benefit of obtaining vitamin $\mathrm{D}$ from skin photosynthesis versus diet or supplementation.

\section{Phosphorus and calcium metabolism New genes}

During the last year, two groups discovered the etiology for hereditary hypophosphatemic rickets with hypercalciuria (HHRH). These two articles were published simultaneously and have expanded our understanding of the mechanism underlying this disease, as well as the physiology of phosphorus homeostasis.

\section{Hereditary hypophosphatemic rickets with hypercalciuria is caused by mutations in the sodium-phosphate cotransporter gene SLC34A3}

Lorenz-Depiereux B, Benet-Pages A, Eckstein G, Tenenbaum-Rakover Y, Wagenstaller J, Tiosano D,

Gershoni-Baruch R, Albers N, Lichtner P, Schnabel D, Hochberg Z, Strom TM

Institute of Human Genetics, GSF National Research Center for Environment and Health, Munich-Neuherberg,

Germany

TimStrom@gsf.de

Am J Hum Genet 2006;78:193-201

Background: Hereditary hypophosphatemic rickets with hypercalciuria (HHRH) is an autosomal recessive form that is characterized by reduced renal phosphate reabsorption, hypophosphatemia, and rickets. It can be distinguished from other forms of hypophosphatemia by increased serum levels of 1,25-dihydroxyvitamin D resulting in hypercalciuria.

Methods: Using SNP array genotyping, we mapped the disease locus in two consanguineous families to the end of the long arm of chromosome 9. The candidate region contained a sodium-phosphate cotransporter gene, SLC34A3, which is expressed in proximal tubulus cells.

Results: Sequencing of this gene revealed disease-associated mutations in five families, including two frameshift and one splice-site mutation. Loss of function of the SLC34A3 protein presumably results in a primary renal tubular defect and is compatible with the HHRH phenotype. It is also shown that the phosphaturic factor FGF23 (fibroblast growth factor 23) is at normal or low-normal serum levels in the patients with $\mathrm{HHRH}$, further supporting a primary renal defect.

Conclusions: Identification of the gene mutated in a further form of hypophosphatemia adds to the understanding of phosphate homeostasis and may help to elucidate the interaction of the proteins involved in this pathway. 


\section{SLC34A3 mutations in patients with hereditary hypophosphatemic rickets with hypercalciuria predict a key role for the sodium-phosphate cotransporter NaPi-Ilc in maintaining phosphate homeostasis}

Bergwitz C, Roslin NM, Tieder M, Loredo-Osti JC, Bastepe M, Abu-Zahra H, Frappier D, Burkett K, Carpenter TO, Anderson D, Garabedian M, Sermet I, Fujiwara TM, Morgan K, Tenenhouse HS, Juppner H

Endocrine Unit, Massachusetts General Hospital and Harvard Medical School, Boston, Mass., USA

cbergwitz@partners.org

Am J Hum Genet 2006;78:179-192

Methods: A genome-wide linkage scan combined with homozygosity mapping was performed, using genomic DNA from a large consanguineous Bedouin kindred that included 10 patients who received the diagnosis of HHRH.

Results: The disease mapped to a 1.6-Mbp region on chromosome 9q34, which contains SLC34A3, the gene encoding the renal sodium-phosphate cotransporter $\mathrm{NaP}(\mathrm{i})$-IIc. Nucleotide sequence analysis revealed a homozygous single-nucleotide deletion (c.228delC) in this candidate gene in all individuals affected by HHRH. This mutation is predicted to truncate the $\mathrm{NaP}(\mathrm{i})$-IIc protein in the first membranespanning domain and thus likely results in a complete loss of function of this protein in individuals homozygous for c.228delC. In addition, compound heterozygous missense and deletion mutations were found in three additional unrelated HHRH kindreds, which supports the conclusion that this disease is caused by SLC34A3 mutations affecting both alleles. Individuals of the investigated kindreds who were heterozygous for a SLC34A3 mutation frequently showed hypercalciuria, often in association with mild hypophosphatemia and/or elevations in 1,25-dihydroxyvitamin D levels.

Conclusion: $\mathrm{NaP}(\mathrm{i})-\mathrm{II} c$ has a key role in the regulation of phosphate homeostasis.

The clinical picture in affected patients with HHRH includes hypophosphatemia, hypercalciuria and nephrocalcinosis, elevated levels of 1,25-OH2D and a bone disease that includes rickets and osteopenia. The clinical picture in heterozygote patients includes hypercalciuria at varying levels without hypophosphatemia, elevated levels of 1,25-OH2D and bone changes such as genua valgus. Three classes of sodium-phosphate cotransporters are expressed in the kidney. They consist of the type I cotransporter, NaPi-I (SLC17A1), and the type II cotransporters, NaPi-Ila (SLC34A1) and NaPi-llc (SLC34A3). Type III transporters, Glvr-1 (SLC20A1) and Ram-1 (SLC20A2), account for $<1 \%$ of the mRNAs encoding the different renal sodium-phosphate cotransporters. The type II cotransporter $\mathrm{NaPi}-$ Ilb (SLC34A2) is not expressed in the kidney but is present in several other tissues, particularly the small intestine, where it is involved in the absorption of dietary phosphate. Both NaPi-lla and $\mathrm{NaPi}-\mathrm{Ilc}$ are regulated in a similar fashion by PTH, FGF23, and dietary phosphate. PTH decreases the abundance of Npt2a protein in the renal brush border membrane. Similarly, renal expression of both cotransporters is reduced by FGF23, whereas mice lacking FGF23 show increased expression of Npt2a, consistent with the renal phosphate retention seen in these animals. Lastly, phosphate deprivation increases Npt2a and Npt2c expression in the proximal tubule. The possibility that mutations in NaPiIla (SLC34A1) cause HHRH was previously excluded. To identify the genetic defect responsible for $\mathrm{HHRH}$, both groups performed genome-wide searches for linkage combined with homozygosity mapping, by using genomic DNA from families with HHRH. Mutations in SLC34A3, the gene encoding for NaPi-llc were found in all affected individuals. The disease has varying degrees of expression. In the heterozygous state, there is a slight loss of phosphorous in the urine that causes low phosphorous levels in the kidney. In contrast with other forms of hypophosphatemic rickets, there is no increase in FGF23 levels and the low phosphorus levels in the kidney increases 1,25 hydroxylase expression and is unopposed by FGF23. The net effect is elevation of 1,25-OH2D resulting in acceleration of intestinal calcium absorption and its urinary excretion. In the homozygous state or in compound heterozygotes, the urinary loss of phosphorus is more significant, resulting in low serum phosphorus and severe rickets (remember, hypophosphatemia interferes with hypertrophic cell apoptosis). A detailed explanation on how hyperphosphatemia causes rickets can be found in another important article published this year [2]. 


\section{Fibroblast growth factor 23 is increased in calcium nephrolithiasis with hypophosphatemia and renal phosphate leak}

Rendina D, Mossetti G, De Filippo G, Cioffi M, Strazzullo P

Department of Clinical and Experimental Medicine, Federico II University Medical School, Naples; Unit of Pediatric Endocrinology, AORN 'G. Rummo', Benevento, and Department of Clinical Pathology, II Medical School, Naples

University, Naples, Italy

J Clin Endocrinol Metab 2006;91:959-963

Background: Nephrolithiasis affects about $10 \%$ of the population in industrialized countries with calcium salts composing more than $80 \%$ of renal stones. A significant percentage of patients with calcium nephrolithiasis and normal parathyroid function show hypophosphatemia and reduced renal phosphate reabsorption (i.e., a renal phosphate leak). The aim of the study was to compare serum levels of fibroblast growth factor 23 (FGF23) in 110 recurrent stone formers with or without renal phosphate leak.

Methods: Six patients affected by X-linked hypophosphatemic rickets, 5 patients affected by oncogenic osteomalacia and 60 unrelated healthy controls. Renal phosphate leak was identified based on the occurrence of idiopathic hypophosphatemia (serum phosphate concentration $<2.50 \mathrm{mg} / \mathrm{dl}$ $(<0.80 \mathrm{mmol} / \mathrm{l}))$, and reduced renal threshold phosphate concentration $(<2.2 \mathrm{mg} / \mathrm{l}(<0.70 \mathrm{mmol} / \mathrm{l}))$.

Results: In 22 stone formers with renal phosphate leak, serum FGF23 concentration was significantly higher as compared with 88 stone formers without renal phosphate leak and with controls $(83.3$ (65.6-101.1) vs. $32.1(26.8-37.4)$ and 24.5 (19.8-29.1) reference units (RU)/ml respectively). Stone formers with renal phosphate leak showed lower FGF23 than patients with oncogenic osteomalacia and X-linked hypophosphatemic rickets (572.3 (235.9-908.7) RU/ml). Among stone formers and controls, serum FGF23 concentration displayed a strong inverse association with serum phosphate $(\mathrm{r}=-0.784$, $\mathrm{p}=0.009)$ and with the rate of tubular phosphate reabsorption $(\mathrm{r}=-0.791, \mathrm{p}=0.008)$.

Conclusions: In this study population, renal phosphate leak affected $20 \%$ of stone formers and was strongly associated with increased serum FGF23 concentration.

In this study cohort, none of the patients exhibited elevated urinary oxalate, urate, or citrate. $20 \%$ of the stone formers had low serum phosphate levels and significantly higher serum FGF23 levels when compared to stone-forming patients without hypophosphatemia and renal phosphate leak or to controls. Although there was no difference in serum calcium, PTH, $25(\mathrm{OH}) \mathrm{D}_{3}$, and $1,25(\mathrm{OH})_{2} \mathrm{D}_{3}$ between stone-forming patients and controls, the stone-forming patients, as a group, had significantly higher urinary calcium levels than the control group. The main difference between these two groups was the renal phosphate reabsorption rate (TmPi/GFR) that was much lower in the patients with phosphate leak. Serum FGF23 levels can differentiate between the patient groups. In the normal control groups the FGF23 range was between 26.8 and $37.4 \mathrm{RU} / \mathrm{ml}$. In the patients with phosphate leak the range was between 65 and $101 \mathrm{RU} / \mathrm{ml}$, whereas in patients with X-linked hypophosphatemic rickets or oncogenic osteomalacia the levels ranged between 258 and $908 \mathrm{RU} / \mathrm{ml}$. Experimental studies have shown that FGF23 downregulates 1,25-hydroxylase in the kidney, resulting in relatively low $1,25(\mathrm{OH})_{2} \mathrm{D}_{3}$, as was expected in the presence of low serum phosphate levels in the kidney. In this study, serum phosphate levels in the patients with urinary phosphate leak were very low and it was expected to see high levels of $1,25(\mathrm{OH})_{2} \mathrm{D}_{3}$ as compared to patients with normal phosphorous levels. However, the high FGF23 levels affected $1,25(\mathrm{OH})_{2} \mathrm{D}_{3}$ levels, indicating that FGF23 levels can affect renal 1,25-hydroxylase activity. The causes for the higher circulating FGF23 levels in the stone-forming patients is unknown, however, there is a possibility of genetic variation affecting the rate of synthesis or the clearance of this molecule. Further studies are necessary to determine the etiology for the elevation in FGF23 serum concentration in these patients. 


\section{The $\beta$-glucuronidase Klotho hydrolyzes and activates the TRPV5 channel}

Chang Q, Hoefs S, van der Kemp AW, Topala CN, Bindels RJ, Hoenderop JG

Department of Physiology, Nijmegen Centre for Molecular Life Sciences, Radboud University Nijmegen Medical Centre, Nijmegen, The Netherlands

Science 2005:310:490-493

Background: Blood calcium concentration is maintained within a narrow range despite large variations in dietary input and body demand. The transient receptor potential ion channel TRPV5 has been implicated in this process.

Methods: TRPV5 knockout mice were studied and were found to have low expression of the klotho gene. Klotho, a type I membrane glycoprotein of $130 \mathrm{kDa}$ is abundantly expressed in mice kidney. Its mRNA expression in other tissues is at least 500 times lower. The study investigated the role of klotho on TRPV5 activity.

Results: TRPV5 is stimulated by the mammalian hormone klotho. Klotho, a $\beta$-glucuronidase, hydrolyzes extracellular sugar residues on TRPV5, entrapping the channel in the plasma membrane. This maintains durable calcium channel activity and membrane calcium permeability in kidney.

Conclusion: Klotho activates a cell surface channel by hydrolysis of its extracellular N-linked oligosaccharides.

Mice lacking TRPV5 display diminished renal calcium reabsorption and severe hypercalciuria, however, due to elevated $1,25(\mathrm{OH})_{2} \mathrm{D}_{3}$, and its activity on TRPV6 expression, compensatory intestinal hyper-reabsorption of dietary calcium is observed. Here we learn that the glycolsylated TRPV 5 is controlled and regulated by the extracellular glucuronidase klotho. TRPV 5 and klotho are both positively regulated by the biosynthesis of vitamin D. Klotho, through its activity, anchors the TRPV 5 molecule to the cell surface and in this way causes accumulation of the calcium channel protein to ensure calcium reabsorption.

\section{Osteoporosis - what we know, how we treat, and future therapeutic modalities}

\section{Pathogenesis of osteoporosis: concepts, conflicts, and prospects}

Raisz LG

University of Connecticut Health Center, Musculoskeletal Institute, Farmington, Conn., USA

raisz@nso.uchc.edu

J Clin Invest 2005;115:3318-3325.

This comprehensive review summarizes the basic pathologic mechanisms that are involved in osteoporosis. The review discusses the central role of estrogen in bone architecture and the importance of calcium and vitamin $\mathrm{D}$ in bone metabolism. The review clearly summarizes the connection between osteoblasts and osteoclasts, and the range of factors that influence this relationship. Understanding the mechanisms of this relationship has an important role in the latest treatment for osteoporosis that is discussed later on in this chapter. 


\section{Calcium plus vitamin $D$ supplementation and the risk of fractures}

Jackson RD, LaCroix AZ, Gass M, Wallace RB, Robbins J, Lewis CE, Bassford T, Beresford SA, Black HR, Blanchette P, Bonds DE, Brunner RL, Brzyski RG, Caan B, Cauley JA, Chlebowski RT, Cummings SR, Granek I, Hays J, Heiss G, Hendrix SL, Howard BV, Hsia J, Hubbell FA, Johnson KC, Judd H, Kotchen JM, Kuller LH, Langer RD, Lasser NL, Limacher MC, Ludlam S, Manson JE, Margolis KL, McGowan J, Ockene JK, O’Sullivan MJ, Phillips L, Prentice RL, Sarto GE, Stefanick ML, Van Horn L, Wactawski-Wende J, Whitlock E, Anderson GL, Assaf AR, Barad D Division of Endocrinology, Ohio State University, Columbus, Ohio, USA jackson.20@osu.edu N Engl J Med 2006;354:669-683

Background: The aim of the study was to assess the efficacy of calcium with vitamin D supplementation for preventing hip and other fractures in healthy postmenopausal women.

Methods: 36,282 postmenopausal women, 50-79 years of age, were recruited. The participants received $1,000 \mathrm{mg}$ of elemental calcium as calcium carbonate with $400 \mathrm{IU}$ of vitamin $\mathrm{D}_{3}$ daily or placebo. Fractures were ascertained for an average follow-up period of 7.0 years.

Results: Hip bone density was $1.06 \%$ higher in the calcium + vitamin D group than in the placebo group $(\mathrm{p}<0.01)$. Intention-to-treat analysis indicated that participants receiving calcium + vitamin $\mathrm{D}$ supplementation had a hazard ratio of 0.88 for hip fracture $(95 \%$ confidence interval (CI) $0.72-1.08)$, 0.90 for clinical spine fracture $(0.74-1.10)$, and 0.96 for total fractures $(0.91-1.02)$. The risk of renal calculi increased with calcium + vitamin D (hazard ratio 1.17; 95\% CI 1.02-1.34). Censoring data from women when they ceased to adhere to the study medication reduced the hazard ratio for hip fracture to $0.71(95 \%$ CI 0.52-0.97). Effects did not vary significantly according to prerandomization serum vitamin D levels.

Conclusions: Among healthy postmenopausal women, calcium with vitamin D supplementation resulted in a small but significant improvement in hip bone density, did not significantly reduce hip fracture, and increased the risk of kidney stones.

This study has been debated and disputed ever since it was published. Although the study group is completely different from our pediatric population with osteoporosis, I chose to include this study for its fundamental importance. It has been the dogma that postmenopausal women require daily supplementation of $1,000 \mathrm{mg}$ of elemental calcium as calcium carbonate and $400 \mathrm{IU}$ of vitamin $\mathrm{D}_{3}$. This study found that this treatment did not decrease the incidence of hip bone fractures, whereas it caused hypercalciuria. In a subgroup of women $>60$, an improvement in hip bone density was associated with a slight decrease in the fracture rate. This study disproves that vitamin D and calcium supplementation are harmless, and shows that treatment with this combination may result in an increase in renal calculi. It shows the difficulty to validate population-based interventions and suggests that interventions should be focused on high-risk individuals. Calcium and vitamin $D$ remain the first line treatment in osteoporosis, though with limited effect. They do, however, have an important role in correcting negative calcium balance. Before we have better means, children at risk should receive calcium and vitamin $D$ supplementation under monitoring of urinary calcium. These results emphasize the importance of further basic and clinical research in osteoporosis in order to provide new modalities of treatment, as presented in the next articles.

\section{New drugs and intervention modalities in osteoporosis}

\section{Recombinant osteoprotegerin for juvenile Paget's disease}

Cundy T, Davidson J, Rutland MD, Stewart C, DePaoli AM

Department of Medicine, Faculty of Medical and Health Sciences, University of Auckland, Auckland, New Zealand N Engl J Med 2005;353:918-923

Background: Juvenile Paget's disease, a genetic bone disease characterized by accelerated bone turnover, results from inactivating mutations in the gene encoding osteoprotegerin - a key regulator of osteoclastogenesis.

Methods: The effects of recombinant osteoprotegerin were investigated in 2 adult siblings with juvenile Paget's disease. 
Results: Bone resorption (assessed by N-telopeptide excretion) was suppressed by once-weekly subcutaneous doses of $0.3-0.4 \mathrm{mg} / \mathrm{kg}$. After 15 months of treatment, radial bone mass increased in 1 patient by $9 \%$ and in the other by $30 \%$; skeletal bisphosphonate retention decreased by 37 and $55 \%$, respectively. These results were accompanied by radiographic improvement. Apart from mild hypocalcemia and hypophosphatemia, no apparent adverse events occurred.

Conclusions: This study confirms the critical role of osteoclastogenesis in regulating bone turnover in humans.

We seem to have a completely new paradigm of osteoporosis treatment. You recall that the interaction between osteoblasts and osteoclasts has a major role in bone metabolism. The osteoblast synthesizes and secretes NF-KB ligand (RANKL) that interacts with its receptor RANK that is located on the osteoclast. The interaction between RANKL and RANK activates the osteoclasts and induces their proliferation and differentiation. At the same time, the osteoblast secretes osteoprotegerin that acts as a decoy receptor for RANKL, and balances RANKL activity. In the absence of osteoprotegerin, as found in juvenile Paget's disease, the equilibrium between osteoblastogenesis and osteoclastogenesis is disturbed, and results in severe accelerated bone turnover and low bone mass. In this study, the authors show that after 8 days of recombinant osteoprotegerin, there was a significant decrease in bone resorption and turnover. Mild hypocalcemia, hyperparathyroidism, and hypophosphatemia were noted in the patients, probably due to the abrupt reduction in bone turnover, but this could be prevented by calcium supplementation. Another new treatment concerning the triangular relationship between RANKL/RANK and osteoprotegerin is presented in the next article.

\section{Denosumab in postmenopausal women with low bone mineral density}

McClung MR, Lewiecki EM, Cohen SB, Bolognese MA, Woodson GC, Moffett AH, Peacock M, Miller PD, Lederman SN, Chesnut CH, Lain D, Kivitz AJ, Holloway DL, Zhang C, Peterson MC, Bekker PJ

Providence Portland Medical Center, Portland, Oreg., USA

mmcclung@orost.com

N Engl J Med 2006;354:821-831

Background: The study investigated the role of denosumab, a fully human monoclonal antibody (IgG2) that binds to RANKL, in postmenopausal women with low bone mineral density.

Methods: 412 postmenopausal women with low bone mineral density ( $\mathrm{T}$ score of -1.8 to -4.0 at the lumbar spine or -1.8 to -3.5 at the proximal femur) were randomly assigned to receive either denosumab every 3 months (at a dose of 6,14 , or $30 \mathrm{mg}$ ), denosumab every 6 months (at a dose of 14, 60, 100 , or $210 \mathrm{mg}$ ), open-label oral alendronate once weekly (at a dose of $70 \mathrm{mg}$ ), or placebo. The percentage change from baseline in bone mineral density in the lumbar spine at 12 months was measured. Results: Denosumab treatment for 12 months resulted in an increase in bone mineral density at the lumbar spine of $3.0-6.7 \%$ (as compared with an increase of $4.6 \%$ with alendronate and a loss of $0.8 \%$ with placebo), at the total hip of $1.9-3.6 \%$ (as compared with an increase of $2.1 \%$ with alendronate and a loss of $0.6 \%$ with placebo), and at the distal third of the radius of $0.4-1.3 \%$ (as compared with decreases of $0.5 \%$ with alendronate and $2.0 \%$ with placebo). Near-maximal reductions in mean levels of serum C-telopeptide from baseline were evident 3 days after the administration of denosumab. The duration of the suppression of bone turnover appeared to be dose-dependent.

Conclusions: In postmenopausal women with low bone mass, denosumab increased bone mineral density and decreased bone resorption. These preliminary data suggest that denosumab might be an effective treatment for osteoporosis.

Denosumab is a human monoclonal antibody (IgG2) that binds to RANKL. In this extensive study, the treatment with denosumab at two different dosages was compared to bisphosphonate alendronate. Denosumab has a rapid onset of action and decreased serum C-telopeptides within 3 days. The observed changes in bone mineral density were at least as great with denosumab as with alendronate, and even greater at the distal third of the radius and total hip. However, it should be kept in mind that RANKL is expressed on cells other than osteoclast precursors, including dendritic cells, $T$ cells and B cells, and is involved not only in osteoclastogenesis but also in functions of the immune system. Interfering with this relationship may disrupt the immune system and expose the patients to infections and/or neoplasms. The paper reports a $1.9 \%$ incidence of neoplasm and a $1 \%$ incidence of unspecified infections in the denosumab group, which was however not statistically significant, but 
certainly needs to be further investigated. The previous hero of anti-osteoporosis treatment was PTH, which gets a new twist in the following article.

\title{
Osteoblast-derived PTHrP is a potent endogenous bone anabolic agent that modifies the therapeutic efficacy of administered PTH 1-34
}

\author{
Miao D, He B, Jiang Y, Kobayashi T, Soroceanu MA, Zhao J, Su H, Tong X, Amizuka N, Gupta A, Genant HK, \\ Kronenberg HM, Goltzman D, Karaplis AC \\ Calcium Research Laboratory and Department of Medicine, Royal Victoria Hospital of McGill University Health \\ Centre, Montreal, Que., Canada \\ J Clin Invest 2005;115:2402-2411
}

Background: The aim of the study was to understand the molecular mechanism that regulates the anabolic effects of PTH on bone density and to assess the variability in the anabolic response to exogenous PTH. Heterozygous mice with targeted disruption of PTHrP, which exhibited impaired bone formation arising from decreased bone mass precursor cell recruitment and increased apoptotic death of osteoblastic cells, were investigated.

Methods: Mice with osteoblast-specific targeted disruption of PTHrP were generated by using Cre-LoxP technology. The defective bone formation was reaffirmed as the underlying etiology.

Results: Daily administration of the 1-34 amino-terminal fragment of parathyroid hormone (PTH 1-34) to $\mathrm{PTHrP}^{+/-}$mice resulted in profound improvement in all parameters of skeletal microarchitecture, surpassing the improvement observed in treated WT littermates.

Conclusions: These findings establish a pivotal role for osteoblast-derived PTH-related protein (PTHrP) as a potent endogenous bone anabolic factor that potentiates bone formation by altering osteoblast recruitment and survival and whose level of expression in the bone microenvironment influences the therapeutic efficacy of exogenous PTH 1-34.

The fundamental approach to the treatment of osteoporosis is the change in equilibrium between the anabolic and resorptive processes in the bone. Although the response to vitamin D and calcium treatment in osteoporosis is very limited, this remains the first line of treatment, and is beneficial for those with a disruption in calcium, vitamin D, and PTH homeostasis. The main treatment options to delay bone resorption are, on the one hand, HRT in women or men (including adolescents with hypogonadism), with the caveats learnt recently, and, on the other hand, bisphosphonates that delay bone resorption processes. RANKL antibodies and osteoprotegerin are now additional futuristic options tackling the resorptive process. As to the anabolic processes of the bone, PTH 1-34 was reported to be effective with a great deal of variability in the response. Surprisingly, its action is enhanced in the heterozygous PTHrP KO as compared to the wild type. In the heterozygous state, treatment with PTH 1-34 resulted in recruitment of more osteoblasts and prevention of their apoptosis. This interaction between treatment and other components of the system raise the question of whether patients who respond better to treatment with PTH 1-34 have lower receptor concentration on their osteoblasts due to polymorphisms? For the pediatric population, treatment with PTH 1-34 is not evaluated nor approved due to the occurrence of osteosarcoma that was found in mice.

\section{New vitamin D analogues for the treatment of osteoporosis}

\section{c-Fos protein as a target of anti-osteoclastogenic action of vitamin $D$, and synthesis of new analogs}

Takasu H, Sugita A, Uchiyama Y, Katagiri N, Okazaki M, Ogata E, Ikeda K

Department of Bone and Joint Disease, Research Institute, National Center for Geriatrics and Gerontology, Obu; Pharmaceutical Research Laboratory, Chugai Pharmaceutical Co. Ltd, Gotemba, and Cancer Institute Hospital, Japanese Foundation for Cancer Research, Tokyo, Japan

J Clin Invest 2006;116:528-535

Background: Although active vitamin D drugs have been used for the treatment of osteoporosis, how the vitamin D receptor (VDR) regulates bone cell function remains largely unknown. The aim of the study was to investigate the role of $1 \alpha, 25$-dihydroxyvitamin $\mathrm{D}_{3}$ treatment in severe osteoporosis. 
Methods: Osteoprotegerin-deficient mice, which exhibit severe osteoporosis due to excessive activation of NF- $\mathrm{B}$ (RANKL/RANK) receptor, were treated with $1 \alpha, 25$-dihydroxyvitamin $\mathrm{D}_{3}$.

Results: $1 \alpha, 25$-Dihydroxyvitamin $\mathrm{D}_{3}$ treatment inhibited bone resorption and prevented bone loss, suggesting that VDR counters RANKL/RANK signaling. $1 \alpha, 25(\mathrm{OH})_{2} \mathrm{D}_{3}$ dose-dependently inhibited MCSF-dependent osteoclast precursor cells differentiation into multinucleate osteoclasts induced by RANKL. Among signaling molecules downstream of RANK, $1 \alpha, 25(\mathrm{OH})_{2} \mathrm{D}_{3}$ inhibited the induction of c-Fos protein downstream RANKL stimulation. Retroviral expression of c-Fos protein abrogated the suppressive effect of $1 \alpha, 25(\mathrm{OH})_{2} \mathrm{D}_{3}$ on osteoclast development. By screening vitamin $\mathrm{D}$ analogs based on their c-Fos-suppressing activity, a new analog named DD281 was found to inhibit bone resorption and prevent bone loss in ovariectomized mice. While DD281 was found to inhibit c-Fos expression more potently than $1 \alpha, 25(\mathrm{OH})_{2} \mathrm{D}_{3}$, it had similar affects on calcium absorption.

Conclusions: c-Fos protein is an important target of the skeletal action of VDR-based drugs, and DD281 is a bone-selective analog that may be useful for the treatment of bone diseases with excessive osteoclastic activity.

In this elegant basic research study the authors illuminate the role of vitamin $\mathrm{D}$ action on osteoclasts. $1,25-\mathrm{OH}_{2} \mathrm{D}$ acts downstream of RANK and inhibits the expression of c-Fos. Many proteins are involved in RANK signaling, but none of them, beside c-Fos, were affected by $1,25-\mathrm{OH}_{2} \mathrm{D}$. Inhibition of the expression of c-Fos results in inhibition of osteoclast recruitment and differentiation. While 1,25$\mathrm{OH}_{2} \mathrm{D}$ inhibits osteoclast differentiation, it promotes differentiation in osteoblasts. Here we learn of a new analog of $1 \alpha, 25(\mathrm{OH})_{2} \mathrm{D}$, DD281, with potent antiresorptive activity on bone relative to its effect on calcium absorption. Further studies are needed to explore whether DD281 is a bone-selective analog that may be useful for the treatment of bone diseases with excessive osteoclastic activity.

\section{Bone mass - new concepts}

\section{Central IL-1 receptor signaling regulates bone growth and mass}

Bajayo A, Goshen I, Feldman S, Csernus V, Iverfeldt K, Shohami E, Yirmiya R, Bab I

Bone Laboratory, Hebrew University of Jerusalem, Jerusalem, Israel

Proc Natl Acad Sci USA 2005;102:12956-12961

Background: Peripheral IL-1 has been implicated as a mediator of the bone loss induced by sex hormone depletion and TNF. The aim of the study was to explore the role of IL-1 in the central nervous system. Methods: The effect on bone of either knockout of the hypothalamic IL-1 receptor type 1 (IL-1RI) or targeted overexpression of human IL-1 receptor antagonist to the central nervous system in mice was investigated.

Results: The IL-1RI-deficient mice (IL-1rKO mice) phenotype revealed low bone mass (LBM), including impairment of bone growth. The process leading to the LBM in both IL-1rKO and IL-1raTG is characterized mainly by doubling the osteoclast number. WT mice express mouse IL-1ra in bone but not in the hypothalamus. Because low levels of IL-1 are present in both tissues, it is suggested that skeletal IL-1 activity is normally suppressed, whereas central IL-1 produces a constant physiologic stimulation of IL-1RI signaling. Either genetic modification in these mice did not decrease testosterone or increase corticosterone serum levels, suggesting that systems other than the gonads and hypothalamo-pituitaryadrenocortical axis mediate the central IL-1RI effect on bone.

Conclusions: Although the pathway connecting the central IL-1RI signaling to bone remodeling remains unknown, the outburst of osteoclastogenesis in its absence suggests that normally it controls bone growth and mass by tonically restraining bone resorption.

The complexity of the hypothalamic influence on bone density is constantly expanding. Some of the hypothalamic input that affects bone density is mediated by leptin and the output is mediated by the sympathetic nervous system modulating osteoblasts. In the previous Yearbook, I mentioned the expression of CART (cocaine-/amphetamine-regulated transcript) in the hypothalamus and its influence on osteoclasts, but the link between CART and osteoclasts is still missing, as no specific 
CART receptor was identified. Previous studies have shown that the cytokine IL-1 has a direct influence on osteoclasts. It induces RANKL expression and directly stimulates the differentiation of osteoclast precursors. In this study the authors show that IL-1 has an important role through the hypothalamic IL-1 receptor on osteoclasts. In the absence of IL-1 activity in the hypothalamus there is an increase in the number of osteoclasts in bones. Receptors for IL-1 are expressed in both bones and the hypothalamus, but peripheral tissues contain specific antagonists to IL-1 receptor. These antagonists are not present in the brain, indicating that the main tone of IL-1 activity on the bone mass may be mediated through the hypothalamic network. More on the brain-bone interaction is presented in the next article.

\section{New concepts - clock genes and osteoblast activity}

\section{The molecular clock mediates leptin-regulated bone formation}

Fu L, Patel MS, Bradley A, Wagner EF, Karsenty G

Department of Molecular and Human Genetics and Bone Disease Program of Texas, Baylor College of Medicine, Houston, Tex., USA

Cell 2005;122:803-815

Background: Mice lacking molecular-clock components (Per and Cry), or lacking Per genes in osteoblasts, display high bone mass, suggesting that bone remodeling may also be subject to circadian regulation. Moreover, Per-deficient mice experience a paradoxical increase in bone mass following leptin intracerebroventricular infusion. Thus, clock genes may mediate the leptin-dependent sympathetic regulation of bone formation. The aim of the study was to determine how clock genes regulate bone mass.

Methods: Mice lacking clock components Per1 and 2 or Cry1 and 2 were investigated.

Results: The sympathetic nervous system regulates the expression of clock genes in osteoblasts and the AP-1 gene. The AP-1 gene expression promotes cyclin D1 expression, osteoblast proliferation, and bone formation. On the other hand, the clock genes mediate the antiproliferative function of sympathetic signaling by inhibiting G1 cyclin expression and downregulating the expression of AP-1 genes. Conclusions: Thus, leptin determines the extent of bone formation by modulating, via sympathetic signaling, osteoblast proliferation through two antagonistic pathways, one of which involves the molecular clock.

The concept that leptin influences bone mass was introduced by Ducy et al. [3], who showed that ob/ob mice have high bone mass and the intracerebroventricular injection of leptin resulted in a reduction of bone mass. We then learnt that the sympathetic system is the link between leptin receptors of the hypothalamus and osteoblasts, through the $\beta_{2}$-adrenergic receptor. In this study the authors investigated the postreceptor $\beta_{2}$-adrenergic cascade in osteoblasts. Bone resorption and bone formation occured in a balanced manner suggesting a circadian rhythm that controls bone mass. The diurnal variation in the synthesis of type I collagen and osteocalcin supports this hypothesis. Most physiological processes in mammals display circadian rhythms that are driven by an endogenous circadian clock. This clock comprises a central component located in the hypothalamic suprachiasmatic nucleus and subordinate clocks in peripheral tissues. Both central and peripheral clocks are operated by positive and negative feedback loops of circadian genes. We now learn that we also have such clock genes in osteoblasts. Upon activation of the $\beta_{2}$-adrenergic receptor in the osteoblast, two intracellular mechanisms are activated. The first involves the osteoblast-specific AP-1 gene, which accelerates osteoblast proliferation and bone formation. The second intracellular arm is composed of non-specific clock genes. Activation of clock genes inhibits the expression of AP-1, which results in the inhibition of osteoblast proliferation and formation. But there is more to the new brain-bone axis. In the last year, two important papers studied the involvement of the cannabinoid receptors, $\mathrm{CB} 1$ and $\mathrm{CB} 2$, on bone density, and explored new aspects of bone mass regulation. 


\title{
Regulation of bone mass, bone loss and osteoclast activity by cannabinoid receptors
}

\author{
Idris Al, van't Hof RJ, Greig IR, Ridge SA, Baker D, Ross RA, Ralston SH
}

Institute of Medical Sciences, University of Aberdeen, Aberdeen, UK

Nat Med 2005:11:774-779

Background: The aim of the study was to investigate the role of the cannabinoid receptors, CB1 and CB2, on bone density.

Methods: The bone density in CB1 knockout mice, ovariectomized CB1 knockout mice, and the role of CB1 and CB2 antagonists were studied.

Results: CB1 knockout mice have increased bone mass and are protected from ovariectomy-induced bone loss. Pharmacological antagonists of CB1 and CB2 receptors prevented ovariectomy-induced bone loss in vivo and caused osteoclast inhibition in vitro by promoting osteoclast apoptosis and inhibiting production of several osteoclast survival factors.

Conclusions: These studies show that the CB1 receptor has a role in the regulation of bone mass and ovariectomy-induced bone loss, and that CB1- and CB2-selective cannabinoid receptor antagonists are a new class of osteoclast inhibitors that may be of value in the treatment of osteoporosis and other bone diseases.

\section{Peripheral cannabinoid receptor, $\mathrm{CB} 2$, regulates bone mass}

Ofek O, Karsak M, Leclerc N, Fogel M, Frenkel B, Wright K, Tam J, Attar-Namdar M, Kram V, Shohami E, Mechoulam R, Zimmer A, Bab I

Bone Laboratory, Hebrew University of Jerusalem, Jerusalem, Israel

Proc Natl Acad Sci USA 2006;103:696-701

Background: The aim of the study was to investigate the role of CB2 receptor on bone mass.

Methods: Mice with a deletion of the CNR2 gene (CB2 ${ }^{-1-}$ mice) were investigated.

Results: CB2 is expressed in osteoblasts, osteocytes, and osteoclasts. CB2-deficient mice phenotype is characterized by increased activity of trabecular osteoblasts (bone-forming cells), increased osteoclast (the bone-resorbing cell) number, and a markedly decreased number of diaphyseal osteoblast precursors. A CB2-specific agonist enhances endocortical osteoblast number and activity and restrains trabecular osteoclastogenesis, apparently by inhibiting proliferation of osteoclast precursors and receptor activator of NF-кB ligand expression in bone marrow-derived osteoblasts/stromal cells. The same agonist attenuates ovariectomy-induced bone loss and markedly stimulates cortical thickness through the respective suppression of osteoclast number and stimulation of endocortical bone formation.

Conclusions: These results demonstrate that the endocannabinoid system is essential for the maintenance of normal bone mass by osteoblastic and osteoclastic CB2 signaling. Hence, CB2 offers a molecular target for the diagnosis and treatment of osteoporosis.

Endogenous cannabinoids bind and activate two G-protein-coupled receptors, a predominantly central cannabinoid receptor type 1 (CB1) and a peripheral cannabinoid receptor type 2 (CB2). CB1 mediates the cannabinoid psychotropic, analgesic, and orectic effects, while CB2 has been implicated recently in the regulation of liver fibrosis and atherosclerosis. The first study investigated the skeletal phenotype in mice with targeted inactivation of $C B 1$ and showed that $C B 1$ KO mice increase their bone mineral density. Moreover, treatment of WT mice with CB1- and CB2-selective antagonists inhibited osteoclast formation and accelerated osteoclast apoptosis. Further trials of the new CB1 antagonist Rimonaband in obese patients will show if this antagonist affects bone mass beyond the reduction of body weight. The second study investigated the role of $\mathrm{CB} 2$ on bone mass by using CB2 $\mathrm{KO}$ and selective antagonists for $\mathrm{CB} 2$. They show that $\mathrm{CB} 2$ signaling has a direct role on osteogenesis and osteoclastogenesis. The CB2 KO mice phenotype showed low bone mass with high turnover that was characterized by low trabecular bone mass and cortical expansion, reminding us of postmenopausal osteoporosis. CB2 receptors were found in osteoblasts, osteocytes, and osteoclasts. A specific CB2 agonist (HU308) was mitogenic in osteoblast lineage and promoted osteoblast differentiation, however, 
it reduced the expression of RANKL mRNA without affecting osteoprotegerin expression. Beside the reduction of RANKL expression, HU308 has an anti-mitogenic effect in osteoclast lineage. Treating ovariectomized mice with HU308 inhibited bone resorption and stimulated endocortical bone formation. These studies raise the possibility for a new therapeutic modality in osteoporosis, a modality that accelerates the osteoblastic arm and reduces the osteoclastogenic arm in bone remodeling.

\section{References}

1. Rodriguez L, Cheng Z, Chen TH, Tu C, Chang W: Extracellular calcium and parathyroid hormone-related peptide signaling modulate the pace of growth plate chondrocyte differentiation. Endocrinology 2005;146:4597-4608.

2. Sabbagh Y, Carpenter TO, Demay MB: Hypophosphatemia leads to rickets by impairing caspase-mediated apoptosis of hypertrophic chondrocytes. Proc Natl Acad Sci USA 2005;102:9637-9642.

3. Ducy P, Amling M, Takeda S, Priemel M, Schilling AF, Beil FT, et al: Leptin inhibits bone formation through a hypothalamic relay: a central control of bone mass. Cell 2000;100:197-207. 